> La prévalence des maladies chroniques du foie ne cesse d'augmenter, du fait de la pandémie de l'obésité. Ces maladies s'étendent de la bégnine stéatose à la stéatopathie non alcoolique (NASH) qui peut évoluer vers le carcinome hépatocellulaire. II n'existe pas de traitement pour ces maladies. La transition stéatose-NASH apparaît déterminante dans leur progression. Au cours de l'obésité, l'activation chronique de la réponse au stress du réticulum endoplasmique (RE) jouerait un rôle crucial dans cette transition, conduisant à la mort cellulaire, à l'inflammation et à l'aggravation des désordres métaboliques. Dans cette revue, nous discutons ces aspects et proposons que le ciblage de cette réponse au stress du RE puisse être pertinent dans la prise en charge thérapeutique de la NASH. <

\section{Les maladies chroniques du foie}

La pandémie de l'obésité et du surpoids constitue un problème mondial de santé publique. En France, plus de $50 \%$ de la population est en surpoids ou obèse ${ }^{1}$ [1]. L'obésité est associée aux maladies chroniques du foie non alcooliques (ou, en anglais, NAFLD pour non alcoholic fatty liver disease) [2] qui affecteraient $25 \%$ de la population mondiale et entre 85 et $98 \%$ des individus obèses morbides. Le spectre des NAFLD s'étend de la stéatose hépatique (ou «foie gras»), à la stéatohépatite (en anglais, NASH pour non alcoholic steato-hepatitis) qui peut progresser vers la cirrhose et le carcinome hépatocellulaire (Figure I). La prévalence de ces pathologies reste sous-estimée en raison de leur évolution asymptomatique.

La stéatose hépatique, de bon pronostic, est caractérisée par une accumulation de lipides au sein des hépatocytes: majoritairement des triglycérides, des diacylglycérols et des céramides, plus minoritaires. La

Vignette (Photo (c) Inserm/Institut clinique de la souris - Éric Robinet). ${ }^{1}$ Enquête 0 b Épi-Roche 2012 et Cohorte épidémiologique Constances 2016.

\section{La réponse au stress du réticulum endoplasmique dans la physiopathologie des maladies chroniques du foie}

Déborah Vallée, Marina Blanc, Cynthia Lebeaupin, Béatrice Bailly-Maitre

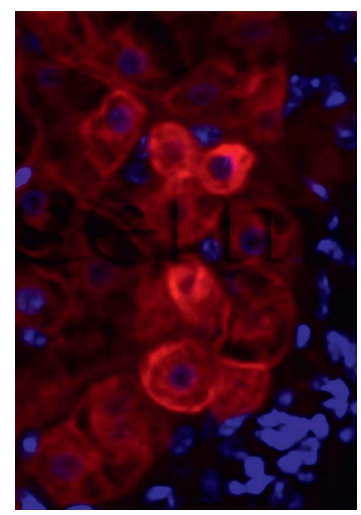

Centre méditerranéen de médecine moléculaire (C3M), Inserm U1065,

151, Route de St Antoine de Ginestière, 06204 Nice, France. beatrice.bailly-maitre@unice.fr

stéatose peut progresser vers la stéatohépatite, au pronostic plus sévère. La stéatohépatite est la forme évolutive de la maladie. Elle est définie par la présence d'une stéatose qui conduit progressivement à l'apparition de lésions inflammatoires, à l'activation de processus de mort hépatocytaire (par apoptose et nécrose), puis au développement de la fibrose. L'ensemble de ces caractéristiques physiopathologiques font de la stéatohépatite un facteur de risque de cirrhose, de carcinome hépatocellulaire ( $\mathrm{CHC}$ ) et d'insuffisance hépatique [3]. La prévalence du CHC est plus élevée chez les patients obèses, avec un risque de décès en lien avec une maladie du foie multiplié par 4,5. Les études épidémiologiques indiquent également une augmentation de la mortalité chez les patients atteints de maladies chroniques du foie.

La transition entre stéatose et NASH repose sur plusieurs facteurs et est déterminante dans la physiopathologie des maladies chroniques du foie. Les mécanismes moléculaires impliqués dans cette transition et l'évolution vers des stades plus sévères restent mal compris. Hormis les mesures hygiéno-diététiques (réduction de la prise alimentaire) et des modifications du style de vie (augmentation de l'activité physique), aucun traitement pharmacologique n'est disponible. II apparaît donc crucial d'élucider les mécanismes participant à la physiopathologie des NAFLD afin de proposer de nouvelles approches thérapeutiques.

Le réticulum endoplasmique $(R \varepsilon)$ est le compartiment cellulaire qui assure le maintien des homéostasies protéique (synthèse, repliement, 


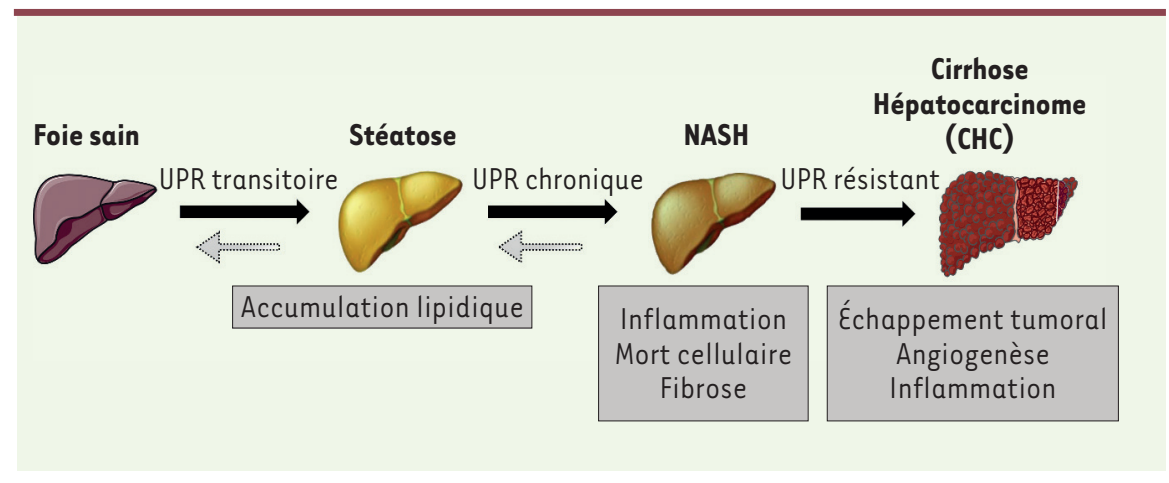

Figure 1. Les complications hépatiques associées à l'obésité. Le foie sain peut évoluer vers la stéatose, un stade bénin, caractérisée par une accumulation de lipides. La stéatose peut progresser vers un stade plus délétère : la stéatohépatite non alcoolique (NASH), caractérisée par l'apparition d'inflammation, de mort cellulaire et de fibrose. La NASH peut conduire à des stades dramatiques tels que la cirrhose et l'hépatocarcinome (CHC).

modifications post-traductionnelles des protéines sécrétées), calcique (stockage du calcium) et lipidique (biogenèse des lipides: phospholipides, cholestérol, triglycérides). L'obésité s'accompagne de perturbations fonctionnelles du RE qui contribuent à l'activation de voies de signalisation qui prennent leurs origines au sein du $R \varepsilon$ et qui sont appelées « réponse au stress du $R E$ ». Cette réponse est impliquée dans la physiopathologie des maladies chroniques du foie. Dans cette revue, nous exposerons les causes de cette réponse et ses conséquences dans la physiopathologie de ces maladies, notamment dans l'apparition de la stéatose et dans la transition vers la NASH.

\section{La réponse au stress du réticulum endoplasmique contribue à la physiologie hépatique}

Le foie assure des fonctions métaboliques et sécrétoires qui sont vitales ainsi que des fonctions de détoxification. II est constitué d'hépatocytes qui représentent environ $80 \%$ des cellules hépatiques. La fraction de cellules restante correspond aux cellules de Kupffer (ou macrophages résidents du foie) et autres cellules immunitaires, aux cellules stellaires (qui sont responsables de la fibrose) et aux cellules endothéliales sinusoïdales. Les hépatocytes sont des cellules sécrétrices qui sont responsables de l'assemblage et de l'export des VLDL (very low density lipoprotein), de la synthèse des protéines plasmatiques (albumine, facteurs de coagulation, protéines de l'inflammation - CRP [protéine C réactive], hepcidine). C'est également dans ces cellules que sont synthétisés les lipides (acides gras, cholestérol, triglycérides), et que sont métabolisés le glucose et les xénobiotiques. Cette myriade de fonctions est assurée par le réticulum endoplasmique de type lisse (siège des enzymes responsables du métabolisme des lipides et des xénobiotiques) et granuleux (siège de la synthèse protéique).

Des modifications de l'environnement extra-hépatique (induits par des virus, des médicaments, une hyperlipidémie ou une inflammation) ou intracellulaires (accumulation de lipides, stress oxydatif) peuvent perturber les fonctions du réticulum endoplasmique. Cela conduit à l'accumulation de protéines mal repliées et enclenche la voie de la réponse au stress du réticulum endoplasmique, une réponse aux protéines mal appariées (UPR, unfolded protein response). La première phase de cette réponse est adaptative: une cascade d'évènements moléculaires est induite afin de réduire la quantité de protéines mal repliées et contrer le stress initial. Ils incluent l) l'inhibition globale de la synthèse protéique, afin d'éviter l'entrée de nouvelles protéines dans la lumière du $R E$; 2) l'augmentation spécifique de la synthèse de protéines chaperonnes et de foldases qui aident au bon repliement des protéines; 3 ) I'activation de processus de dégradation des protéines non conformes qui soit dépend du RE (le système ERAD, endoplasmic-reticulum-associated protein degradation) soit en est indépendant (par autophagie). Si la cellule ne parvient pas à remédier aux conséquences du stress initial (stress intense ou chronique), les mêmes voies moléculaires conduiront alors, dans une seconde phase, à la mort cellulaire par apoptose (Figure 2).

\section{Les acteurs de la réponse au stress}

Trois protéines transmembranaires du réticulum endoplasmique participent comme senseur à sa réponse au stress : IREl $\alpha$ (inositol-required endoribonuclease $1 \alpha$ ), une sérine/thréonine kinase dotée d'une activité endoribonucléasique; la kinase PERK (PKR-like ER kinase); et la protéine ATF6 (activating-transcription factor 6) [4]. En l'absence de protéines mal conformées, IREl $\alpha$, PERK et ATF6 sont maintenues inactives par la protéine chaperonne BiP (binding immunoglobulin protein), également connue sous le nom de GRP78 (78-kDa glucose regulated protein). Lorsque la mauvaise conformation des protéines devient trop importante, GRP78 se dissocie des trois senseurs afin d'aider au bon repliement des protéines, ce qui conduit à l'homodimérisation et à l'autophosphorylation de deux d'entre eux, les kinases IREl $\alpha$ et PERK, et à l'activation des trois mécanismes de l'UPR.

\section{IREl $\alpha$}

La voie reposant sur IREl $\alpha$ est la plus conservée des trois voies de I'UPR au cours de l'évolution. Cette protéine présente deux activités enzymatiques: endoribonucléasique (RNase) et, dans son domaine cytosolique, sérine/thréonine kinase. Son activité endoribonucléa- 


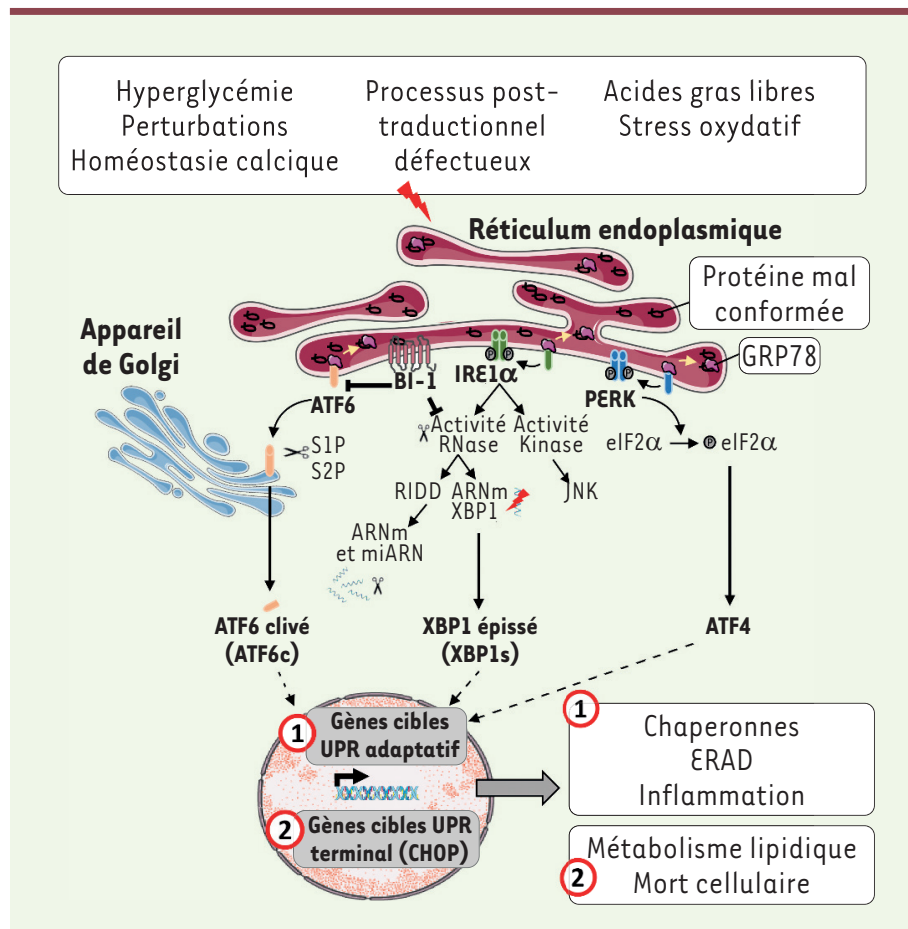

Figure 2. La réponse au stress du réticulum endoplasmique. La réponse au stress du réticulum endoplasmique (RE) est transmise par trois protéines effectrices transmembranaires: IREl $\alpha$ (inositol-requiring enzyme $1 \alpha$ ), PERK (protein kinase r-like endoplasmic reticulum kinase) et ATF6 (activating transcription factor 6). À l'état basal, ces protéines sont maintenues inactives par leur interaction avec la GRP78 (glucose-regulated protein 78). Lors d'un stress, la GRP78 se dissocie de ces trois protéines, conduisant à leur activation. PERK et IREl $\alpha$ se dimérisent et s'auto-phosphorylent. IREl $\alpha$ possède à la fois une activité kinase et endoribonucléase (RNase) conduisant à la production du facteur de transcription XBPls (Xbox-binding protein 1). PERK phosphoryle elF2 $\alpha$ (eukaryotic initiation factor 2), limitant ainsi la traduction protéique et favorisant la traduction du facteur de transcription ATF4 (activating-transcription factor 4). ATF6 migre dans le Golgi où il sera clivé, aboutissant à la production d'un facteur de transcription : ATF6 clivé. Dans un premier temps, ces trois facteurs de transcription (ATF6c, XBP1s, ATF4) visent à rétablir de façon coordonnée l'homéostasie du RE (synthèse de chaperonnes, augmentation de la dégradation des protéines du RE via ERAD [endoplasmic reticulum-associated degradation]). Néanmoins, si le stress est chronique ou intense, les mêmes voies enclenchent la mort de la cellule par apoptose, notamment via le facteur de transcription CHOP (C/EBP homologous protein) et les kinases JNK (c-Jun $N$-terminal kinases).

sique permet un épissage particulier (excisant 26 nucléotides) de I'ARN messager (ARNm) codant XBPls, un facteur de transcription clef des gènes de la réponse UPR et de la lipogenèse. Cette activité RNase d'IREl $\alpha$ est également à l'origine de la dégradation spécifique d'ARNm et de microARN impliqués dans de nombreuses fonctions cellulaires (mort cellulaire, métabolisme, cancer) par un mécanisme appelé RIDD (regulated-IREI-dependent decay) [5] $(\rightarrow)$.
IREl $\alpha$ peut également s'associer à de nombreux partenaires par son domaine cytosolique pour induire une cascade d'activation de protéines kinases. TRAF2 (TNF receptor-associated factor), une protéine adaptatrice, s'associe en effet au domaine kinase d'IREl $\alpha$, formant un complexe (IREl $\alpha /$ TRAF2) qui interagit avec ASK1 (apoptosis signal-regulating kinase 1), une protéine impliquée dans la réponse inflammatoire, qui active NFKB (nuclear factor-kappa B) en phosphorylant son inhibiteur $(\mid \kappa B)$, et dans l'apoptose en activant la kinase JNK (c-Jun $\mathrm{N}$-terminal kinase).

\section{PERK}

PERK est une protéine transmembranaire du RE qui comporte un domaine senseur de stress, localisé dans la lumière du $\mathrm{RE}$ (en position $\mathrm{N}$-terminale), et un domaine kinase (en position C-terminale). Activée, PERK phosphoryle elF2 $\alpha$ (eukaryotic translation initiation factor 2, subunit) sur sa sérine 51 . Ceci conduit à l'arrêt de la traduction des ARNm par empêchement de l'assemblage des ribosomes 805 , et ainsi à la diminution de la synthèse protéique. Paradoxalement, cette voie permet néanmoins la synthèse d'ARNm spécifiques qui possèdent un IRES (internal ribosomal entry site) ou présentent un petit UORF (upstream open reading frame) dans leur région 5'UTR (5' untranslated region), comme I'ARNm codant ATF4 (activating-transcription factor 4), un facteur de transcription clef impliqué dans la réponse UPR. ATF4 active les gènes de I'UPR « adaptatif » dont les produits participent à l'aide au repliement des protéines (gènes codant les chaperonnes), à leur élimination si nécessaire (gènes de l'autophagie), au maintien de l'homéostasie d'oxydo-réduction - induction d'une forte réponse anti-oxydante via la phosphorylation d'un autre substrat de PERK, NRF2 (nuclear factor [erythroid-derived 2]-like 2) [6] - et au métabolisme des acides aminés [7]. Si le stress devient chronique ou intense (UPR chronique), ATF4 favorise l'expression du facteur de transcription pro-apoptotique CHOP (C/EBP homologous protein), qui augmentera l'expression des membres pro-apoptotiques de la famille $\mathrm{Bcl}-2$ ( $B$-cell lymphoma 2) et conduira à l'apoptose de la cellule.

\section{ATF6}

ATF6 est une protéine transmembranaire dont la partie cytosolique est un facteur de transcription possédant un domaine BZIP. II existe deux isoformes d'ATF6: ATF $6 \alpha$, exprimé de façon ubiquitaire, et ATF6 $\beta$ présent uniquement au niveau intestinal. À la suite d'un stress, ATF6 se localise au niveau de l'appareil de Golgi dans lequel il est clivé par les protéases résidentes S1P et S2P (site-1/2-protease), libérant un fragment cytosolique 
«ATF6 clivé » qui, migrant vers le noyau, régule la transcription des gènes de l'UPR [8]. ATF6 clivé ne module pas seulement l'expression des gènes impliqués dans le repliement des protéines et l'ERAD, il peut également activer la transcription de CHOP, induisant alors l'apoptose de la cellule. ATF6 clivé active également l'expression d'XBPI non épissé, communiquant ainsi avec la voie IREl $\alpha$.

Des études réalisées chez la souris indiquent que la voie IREl $\alpha$ est essentielle au développement du foie: la délétion du gène codant $\mid R \varepsilon l \alpha$ conduit à une létalité embryonnaire due à une hypoplasie hépatique. Bien que PERK et ATF6 $\alpha$ ne soient pas indispensables pour le développement du foie, les souris dont les gènes codant PERK et ATF6 $\alpha$ ont été invalidés, ainsi que les souris dont le gène codant IREl $\alpha$ a été invalidé spécifiquement au niveau hépatocytaire, sont incapables de résister à un stress hépatique aigu induit par une injection de tunicamycine ou à un stress chronique par exposition à un régime gras [9]. Ces souris développent des maladies hépatiques, ce qui suggère un rôle majeur de ces trois voies de I'UPR dans le maintien de l'homéostasie hépatique.

\section{Le rôle ambivalent de l'UPR dans la physiopathologie des NAFLD : ami ou ennemi ?}

La durée et l'intensité de la réponse UPR apparaît déterminante dans l'engagement des mécanismes d'abord protecteurs et qui progressent ensuite vers une réponse qui s'avère délétère. La réponse UPR transitoire est en effet un mécanisme protecteur, gardien de la physiologie hépatique. Elle protège les hépatocytes de l'exposition excessive aux nutriments, aux xénobiotiques ainsi qu'aux stress intracellulaires. Une activation transitoire de l'UPR est ainsi observée en situation postprandiale. L'activation transitoire du RIDD d'IREl $\alpha$ conduit également à la dégradation des ARNm codant pour deux cytochromes P450 responsables de la métabolisation de l'acétaminophène en un métabolite cytotoxique pour le foie. Le RIDD réprime aussi les miR-34 et miR200 , ce qui protège l'homme et la souris exposés à un régime gras de l'apparition de la stéatose [10].

Le foie est donc protégé par cette réponse UPR transitoire protectrice. Son activation chronique contribuera à la transition de la stéatose vers la NASH, en favorisant les désordres inflammatoires, métaboliques et les processus de mort cellulaire [11] $(\rightarrow)$.

$\rightarrow$ Voir la Synthèse de F. Foufelle et P. Ferré, $m / s n^{\circ} 3$, mars 2007, page 291

Son activation soutenue (UPR résistant) contribuerait, elle, à la progression des NAFLD vers les stades dramatiques de la maladie hépatique (cirrhose, CHC) (Figure I).

Le premier lien entre l'obésité, l'activation du stress du RE et l'apparition de la stéatose fut mis en évidence par l'équipe du Dr Hotamisligil à Harvard, qui rapporta une augmentation des marqueurs de stress du $R \varepsilon$ au sein de foies stéatosiques isolés à partir de souris génétiquement obèses (ob/ob et $d b / d b$ ) ou rendues obèses par un régime alimentaire riche en graisses (HFD pour high fat diet) [12]. L'activation de I'UPR a également été observée chez les patients présentant une stéatose. Les marqueurs de I'UPR sont en effet amplifiés dans les foies stéatosiques de patients obèses. Leur diminution est corrélée à la perte de poids des patients ainsi qu'à la réduction de la teneur en lipides hépatiques consécutives à une chirurgie bariatrique [13]. L'activation de I'UPR est également observée dans les foies de patients obèses atteints de NASH. II en est de même chez des souris nourries avec un régime déficient en méthionine et choline (MCD) qui présentent les caractéristiques physio-pathologiques de la NASH, mais sans obésité [14], et chez des souris nourries avec des régimes $\mathrm{HFD} /$ fructose ou $\mathrm{HFD} /$ sans choline qui présentent les caractéristiques de la NASH et de l'obésité. Une activation directe de la voie IREl $\alpha-X B P l$ a d'autre part été observée in vivo au cours du développement de la résistance à l'insuline dans le foie [15]. De plus, la surexpression hépatique des chaperonnes résidentes du RE, comme GRP78/BiP (78-kDa glucose-regulated protein), améliore la sensibilité à l'insuline et normalise la stéatose chez les souris obèses [16] en inhibant le stress $\mathrm{du} R \varepsilon$, ce qui apporte une preuve directe que le stress du $R \varepsilon$ dans le foie contribue au développement des NAFLD associées à l'obésité.

\section{Le rôle des lipides dans la mise en place de la réponse UPR}

En situation d'obésité, l'activation chronique de la réponse UPR pourrait être enclenchée par des stress à la fois environnementaux extra-hépatiques (acides gras libres, céramides, cytokines) et intracellulaires, tels que l'accumulation de lipides per se au sein des hépatocytes, une caractéristique physiopathologique des NAFLD. Bien que les triglycérides soient la catégorie de lipides la plus abondante, les analyses lipidomiques ont révélé la présence de diacylglycérols et de céramides dans des biopsies de foies humains stéatosiques [17]. Ces lipides inhiberaient la voie de signalisation de l'insuline hépatique, contribuant à l'apparition de la résistance à l'insuline et du stress du RE. Savoir si la résistance à l'insuline hépatique ou la stéatose se développent en premier reste débattu. II est cependant établi que la résistance à l'insuline est un facteur de risque dans la progression des NAFLD [18].

L'accumulation anormale de lipides dans les hépatocytes perturberait l'homéostasie du calcium $\left(\mathrm{Ca}^{2+}\right)$ dans le RE, activant chroniquement le stress du RE dans le foie. En effet, chez les souris obèses, l'accumulation excessive de lipides dans le foie entraîne un déséquilibre dans la composition lipidique de la membrane du $R \varepsilon$ avec un rapport plus élevé de phosphatidylcholine $(P C)$ par rapport à la phosphatidyléthanolamine (PE), altérant la fluidité de la membrane. Ce changement de ratio $P C / P \varepsilon$ est à l'origine d'une diminution de l'activité de SERCA (sarco/endoplasmic reticulum $\mathrm{Ca}^{2+}$ ATPase), 


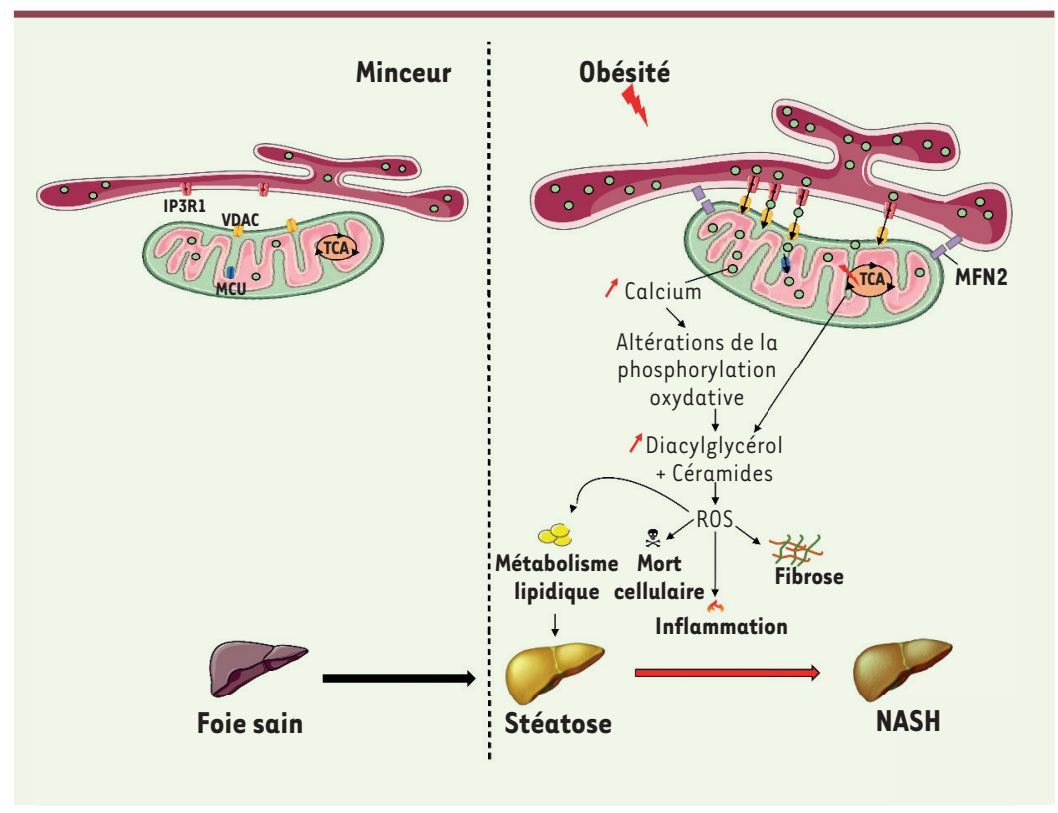

Figure 3. Les MAM, un lieu d'échange entre le réticulum endoplasmique et la mitochondrie. Le réticulum endoplasmique peut former des structures d'échanges avec la mitochondrie, nommées MAM (mitochondria-associated membrane) permettant le passage du calcium $\left(\mathrm{Ca}^{2+}\right)$ et d'espèces lipidiques de la lumière du $R E$ vers la mitochondrie. Au cours de l'obésité, le nombre de MAM augmente, surchargeant la mitochondrie en calcium. Cette surcharge altère les fonctions mitochondriales, engendrant un défaut de phosphorylation oxydative et du cycle d'acide tricarboxylique, conduisant à la production d'espèces réactives de l'oxygène (ROS), I'apparition de la stéatose puis sa transition vers la NASH via l'induction d'inflammation, de mort cellulaire et de fibrose.

la pompe qui permet l'entrée du $\mathrm{Ca}^{2+}$ dans le RE. II s'ensuit une réduction de la concentration de calcium dans le $R \varepsilon$, une accumulation de protéines mal repliées, puisque la majorité des chaperonnes sont dépendantes du $\mathrm{Ca}^{2+}$, et, par conséquent, l'induction de la réponse UPR. Réciproquement, la surexpression de SERCA ou la correction de la composition des membranes en phospholipides dans le $R E$ réduit ce stress et corrige la stéatose chez des animaux obèses. Ainsi, les perturbations lipidiques et de I'homéostasie calcique seraient promotrices du stress du RE hépatique dans l'obésité [19] $(\rightarrow)$. $\rightarrow$ Voir la Nouvelle de M. Flamment et F. Foufelle, $m / s n^{\circ} 1$, janvier 2012, page 13 Comment l'obésité altère l'activité de la pompe SERCA, reste cependant à explorer. Une récente étude suggère que les lipides peuvent induire directement un stress du RE par l'intermédiaire des senseurs IREl $\alpha$ et PERK qui pourraient agir comme des capteurs détectant via leur domaine transmembranaire, les caractéristiques biophysiques des membranes lipidiques reposant sur le rapport des chaînes acyle insaturées et saturées qui affectent la fluidité et l'épaisseur de la membrane [20].

Le RE établit des zones de contacts étroits avec les mitochondries: les membranes associées aux mitochondries (MAM) (Figure 3), qui permettent le transfert de $\mathrm{Ca}^{2+}$ et d'espèces lipidiques entre les 2 organelles. Ainsi, au sein de la membrane du RE, le récepteur de l'inositol triphosphate, IP3R, participe à la libération passive de $\mathrm{Ca}^{2+}$, permettant à la mitochondrie de se charger en calcium d'abord via le canal VDAC (voltage-dependent anion-selective channel 1) présent sur sa membrane externe, puis via le canal MCU (mitochondrial calcium uniporter) localisé sur sa membrane interne. En situation d'obésité, le nombre de MAM augmente, surchargeant les mitochondries en $\mathrm{Ca}^{2+}$ et altérant leurs fonctions, notamment la phosphorylation oxydative. Cette altération de la phosphorylation oxydative est associée à l'apparition de la stéatose, à un dysfonctionnement du cycle d'acide tricarboxylique, à l'accumulation d'intermédiaires lipidiques toxiques (céramides, diacylglycérols) conduisant à la production de ROS (reac- tive oxygen species), à l'apparition de l'inflammation, de la fibrose et à la transition stéatose-NASH [21]. Les lipides jouent ainsi un rôle essentiel dans la mise en place de la réponse UPR et de la stéatose, puis dans son maintien vers des stades délétères.

\section{La réponse UPR chronique favorise la transition stéatose-stéatohépatite}

Suite à l'établissement de la stéatose qui vulnérabilise les hépatocytes, la mise en place de I'UPR «chronique » favoriserait la transition vers la stéatohépatite en aggravant les désordres métaboliques et en induisant des processus inflammatoires, de mort cellulaire et de la fibrose, les caractéristiques physiopathologiques de la stéatohépatite. Les patients atteints de NASH présentent en effet une inflammation qui est corrélée à la gravité histologique de leurs atteintes hépatiques [22]. Les biopsies de foie de ces patients révèlent également un nombre significativement plus élevé d'hépatocytes en apoptose (positifs au marquage TUNEL [terminal deoxynucleotidyl transferase dUTP nick end labeling], activation des caspases exécutrices de l'apoptose - caspases-3 et - 7 - et augmentation de l'expression du récepteur de mort Fas) par rapport aux foies de patients minces ou stéatosiques [23].

\section{La réponse inflammatoire dépendante du stress du RE dans la NASH}

\section{Le stress du RE active les voies NFKB et JNK}

Lors d'un stress chronique, IREl $\alpha$ activée peut former un complexe avec TRAF2 activant les protéines kinases 


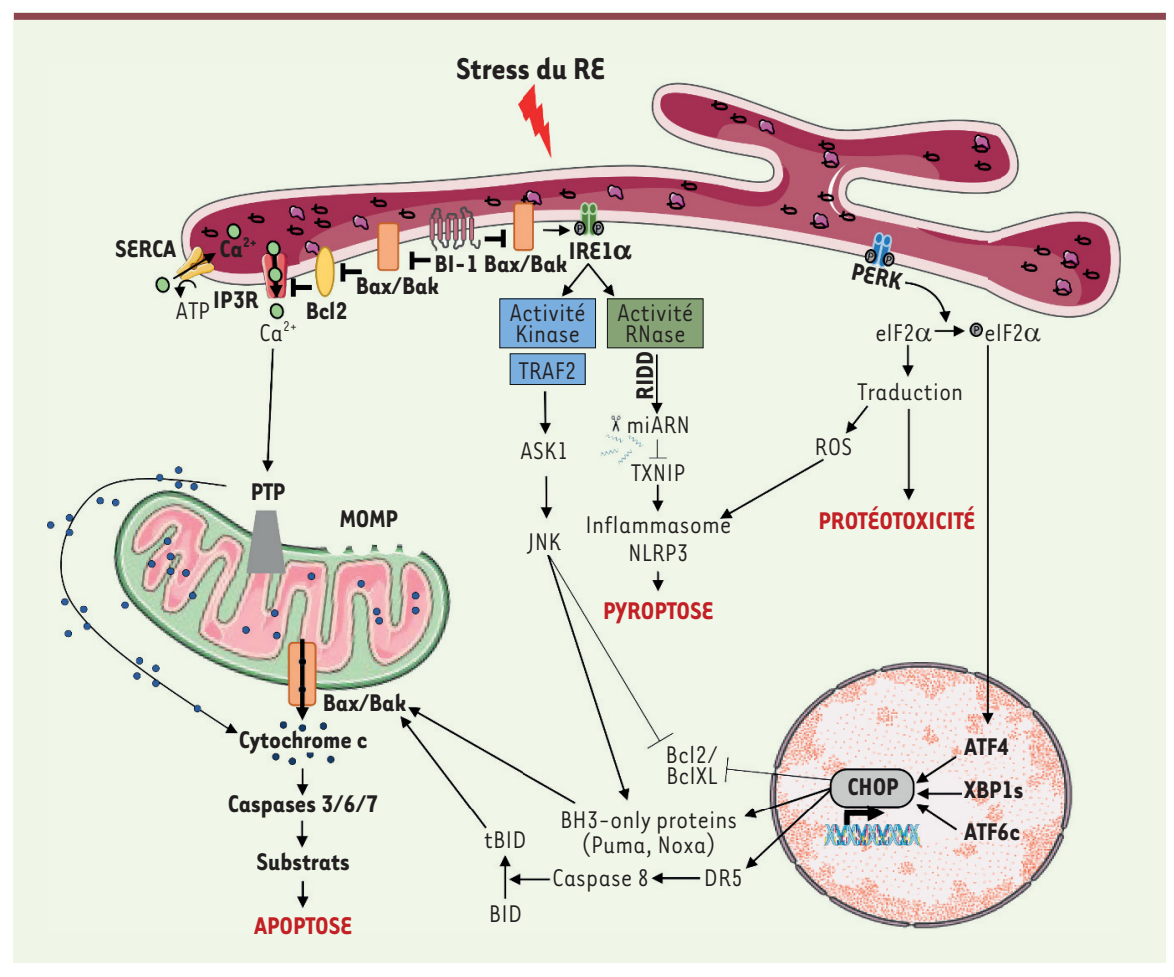

Figure 4. Mécanismes de mort cellulaire dépendants du stress du réticulum endoplasmique. Lors d'un stress au niveau du réticulum endoplasmique ( $R \varepsilon)$, un flux calcique de la lumière du RE vers la mitochondrie intervient, entrainant l'ouverture du canal PTP (permeability transition pore) mitochondrial, une dérégulation $\mathrm{du}$ potentiel membranaire mitochondrial et une perméabilisation de la mitochondrie au niveau de sa membrane externe MOMP (mitochondrial outer membrane permeabilization). Ces dérégulations aboutissent à la libération du cytochrome c, l'activation de la caspase-9, puis des caspases-3/ -6/ -7 et à l'apoptose. Les protéines anti- et pro-apoptotiques régulent également la libération du cytochrome $c$ au niveau de la mitochondrie, inhibant ou favorisant l'induction de l'apoptose. Bcl2 (B-cell lymphoma 2), BclXL (B-cell lymphoma extra large), $\mathrm{Bax}(\mathrm{BCl}-2$-associated $X$ protein) et Bak ( $\mathrm{BCl}-2$ homolo-

gous antagonist killer) peuvent aussi être ancrés au niveau du réticulum endoplasmique où ils régulent la libération de calcium. L'activation des voies IREl $\alpha$ (inositol-requiring enzyme $1 \alpha$ ), PERK (protein kinase $R$-like endoplasmic reticulum kinase) et ATF6 (activating-transcription factor 6) conduit à l'accumulation d'un facteur de transcription pro-apoptotique CHOP (C/EBP homologous protein), qui augmente l'expression des protéines pro-apoptotiques BH3-only proteins (PUMA [p53-upregulated modulator of apoptosis], NOXA [phorbol-12-myristate-13-acetate-induced protein], BIM [BCl-2 interacting mediator of cell]) et diminue l'expression des protéines anti-apoptotiques $\mathrm{Bcl} 2 \mathrm{et} \mathrm{Bcl}-\mathrm{XL}$.

impliquées dans l'inflammation. Le complexe IREl $\alpha$ /TRAF2 active ASK1 (apoptosis signal-regulating kinase 1), puis NFKB et les kinases de la famille JNK impliquées dans l'activation transcriptionnelle de voies pro-inflammatoires et pro-apoptotiques (Figure 4). L'activation de NFKB conduit dans un premier temps à l'induction de réponses inflammatoires qui favorisent la survie des cellules. Mais, au cours de la NASH, NFKB favorise ensuite les lésions hépatiques, la fibrose et l'évolution vers le CHC. L'activation de PERK réduit également la traduction d'IKB, renforçant l'activité de NFKB [24].

Chez le patient obèse, la perméabilité intestinale est augmentée, ce qui favorise le passage dans le sang de composés bactériens comme le lipopolysaccharide (LPS), augmentant leurs niveaux circulants (endotoxémie). Dans les hépatocytes, le LPS activerait via $N F \kappa B$ une réponse pro-inflammatoire « à bas bruit » et à visée anti-apoptotique. Cette activité légèrement augmentée de NFKB contribuerait à la protection des hépatocytes. Néanmoins, au-delà d'un certain seuil, elle serait à l'origine de la sécrétion de facteurs inflammatoires et chimiotactiques, participant ainsi à l'aggravation de l'inflammation hépatique, l'induction de l'apoptose et l'apparition de fibrose [25]. Dans les foies de souris soumises à un régime déficient en méthionine et choline (induisant la NASH), NFKB serait un médiateur important qui participe au développement des lésions de la NASH. Dans des hépatocytes primaires humains exposés aux acides gras saturés, l'activation de la signalisation NFKB par CHOP (CCAAT/ enhancer-binding protein $[C / \varepsilon B P]$ homologous protein) serait un mécanisme impliqué dans la progression de la stéatose vers la NASH, entraînant la sécrétion de cytokines impliquées dans la mort cellulaire et l'inflammation [26].

\section{Stress du RE et production de protéines de la phase aiguë de l'inflammation}

L'augmentation de la sécrétion de protéines de phase aiguë par les hépatocytes (comme la CRP [C-reactive protein]) est un indicateur d'inflammation hépatique qui peut être associé au stress du RE. CREBH ( $C$-AMP response element-binding protein) est un facteur de transcription impliqué dans la phase aiguë de l'inflammation qui est exprimé uniquement dans le foie. Suite à un stress du RE dans les hépatocytes, CREBH subit une protéolyse intra-membranaire permettant la transactivation des gènes de l'UPR et des gènes des protéines inflammatoires. Ainsi, comparés aux patients atteints de stéatose, les patients NASH présentent des taux de 
CRP sériques significativement plus élevés [27]. L’hepcidine, une hormone peptidique sécrétée par le foie en réponse au stress du RE et à l'inflammation, également induite par CREBH, régule l'homéostasie du fer et une surcharge en fer a été observée chez les patients présentant une NASH avec un risque accru d'aggravation vers la fibrose. Les cytokines pro-inflammatoires, telles que le TNF $\alpha$ (tumor necrosis factor alpha) ou I'IL-6 (interleukine-6), ou encore le LPS, peuvent induire l'expression de CREBH, perpétuant ainsi un état inflammatoire lors d'un stress du RE.

\section{Le rôle de l'inflammasome}

Les inflammasomes sont des complexes multi-protéiques qui détectent des signaux de danger et conduisent à la sécrétion de cytokines proinflammatoires lors de stress cellulaires. L'inflammasome NLRP3 (NODlike receptor family, pyrin domain-containing 3), l'un des plus étudiés, est activé dans les foies de patients et de souris NASH [28]. Son activation nécessite deux signaux successifs : un premier, qui induit la transcription et la traduction des cytokines pro-IL-1 $\beta$ et pro-IL-18; et un second, qui sert de médiateur à son assemblage avec la protéine adaptatrice ASC (apoptosis-associated speck-like protein containing a CARD) et les caspases- 1 et -11 . Le complexe ainsi formé permettra I'activation des caspases qui y sont associées, conduisant au clivage des pro-cytokines en cytokines IL-1 $\beta$ et IL-18, qui sont des médiateurs pyrogéniques, déclenchant la réponse inflammatoire. L'inflammasome NLRP3 joue un rôle important dans l'apparition de la stéatose. Les souris déficientes pour la caspase-1, la caspase-11, NLRP3 ou ASC (incapables de produire les cytokines) soumises à un régime riche en graisses sont protégées de la stéatose et de la résistance à l'insuline [29]. L'activation soutenue de NLRP3 conduit à la mort cellulaire par pyroptose, une mort cellulaire programmée dépendant de la caspase-1 (ou -11) et conduisant à un gonflement cellulaire, à la formation de pores dans la membrane, par clivage (en $\mathrm{N}$-terminal) de la gasdermine $\mathrm{D}$ en un fragment de $31 \mathrm{kDa}$ (gasdermine D-N) à l'origine de cette formation de pores, favorisant la libération de I'IL-1 $\beta$ et de I'IL-18 [30]. Les souris déficientes en gasdermine $D$ sont résistantes à la NASH et, inversement, la surexpression de la gasdermine D favorise la NASH, chez la souris saine.

Les souris qui expriment une forme constitutivement active de NLRP3 développent rapidement une NASH. Ces souris présentent une augmentation de la mort hépatocytaire par pyroptose, une aggravation de I'inflammation hépatique, une forte activation des cellules stellaires et de la fibrose [31]. Au contraire, l'inhibition de NLRP3 par traitement pharmacologique améliore la physiopathologie des NAFLD et la fibrose chez les souris diabétiques et obèses [32]. De nombreuses études ont établi un lien entre le stress du RE et l'activation de NLRP3 [28]. Ainsi, chez les souris génétiquement obèses, une endotoxémie ${ }^{2}$ qui accentue les activités hépatiques d'IREl $\alpha$ et de PERK, conduit à la surexpression de CHOP, induisant l'expression à la fois des caspases-1 et -11 et de PUMA (p53-upregulated modulator of apoptosis), un membre pro-apoptotique de la famille $\mathrm{Bcl}-2$ déclenchant la pyroptose et

${ }^{2}$ Présence d'endotoxine (LPS) dans le sang. l'apoptose hépatocytaire. L'acide tauroursodéoxycholique (TUDCA), qui inhibe le stress du RE, protège contre cette activation de NLRP3 dépendant des caspases et d'IREl $\alpha$ et de PERK [28].

L'inflammasome peut également être activé indirectement par IREl $\alpha$ et son activité RIDD capable de dégrader le miARN miR-17 qui réprime TXNIP (thioredoxin-interacting protein). TXNIP se lie et active NLRP3. L'accroissement de son expression contribue ainsi à l'activation de l'inflammasome, augmentant la réponse inflammatoire et favorisant la mort cellulaire [33]. TXNIP favorise aussi la production des ROS, qui contribuent également à l'activation de l'inflammasome. De même, la sur-activation de la fonction RNase d'IREl $\alpha$, chez des souris déficientes pour BI-1 (Bax inhibitor 1) l'inhibiteur endogène de l'activité RNase d'IREl $\alpha$, soumises à un régime riche en graisses, conduit à la NASH en lien avec une augmentation de l'expression hépatique de TXNIP [34], à I'activation de I'inflammasome et de la pyroptose dans les hépatocytes. L'inflammation et la mort cellulaire dépendant de l'inflammasome (pyroptose, apoptose) jouent un rôle central dans la progression de la stéatose vers la NASH.

\section{Stress du RE, production de ROS et inflammation}

Le stress oxydatif, causé par la surproduction de ROS et de radicaux libres, contribuerait au développement des NAFLD. Au cours de la stéatose, l'accumulation d'acides gras et la surcharge en $\mathrm{Ca}^{2+}$ altèrent la fonction des mitochondries (oxydation et respiration) entraînant une augmentation de la production de ROS. Des voies oxydatives alternatives sont alors activées dans les peroxysomes ( $\beta$-oxydation) et les microsomes ( $\omega$-oxydation), amplifiant les niveaux de radicaux libres, ce qui altère l'oxydation des acides gras, augmente la production de glycérol-3-phosphate et la lipogenèse, aggravant la stéatose [35]. Le stress oxydatif pourrait agir comme un déclencheur dans la transition stéatose-NASH. La réponse anti-oxydante est orchestrée par le facteur nucléaire NRF2, fortement exprimé dans les organes détoxifiants comme le foie. Suite à un stress du RE, PERK phosphoryle NRF2 et le stabilise, ce qui permet de réduire les niveaux élevés de ROS [6]. L'activation de la réponse au stress du RE, jouerait ainsi, via NRF2, un rôle cytoprotecteur vis-àvis de l'inflammation induite par le stress oxydatif. Les souris déficientes pour NRF2 sous régime déficient en méthionine et choline, présentent une aggravation des caractéristiques de la NASH et de l'inflammation, résultant d'une augmentation significative du stress oxydatif par rapport à des souris sauvages. Inversement, une activation soutenue de NRF2 protège les souris 


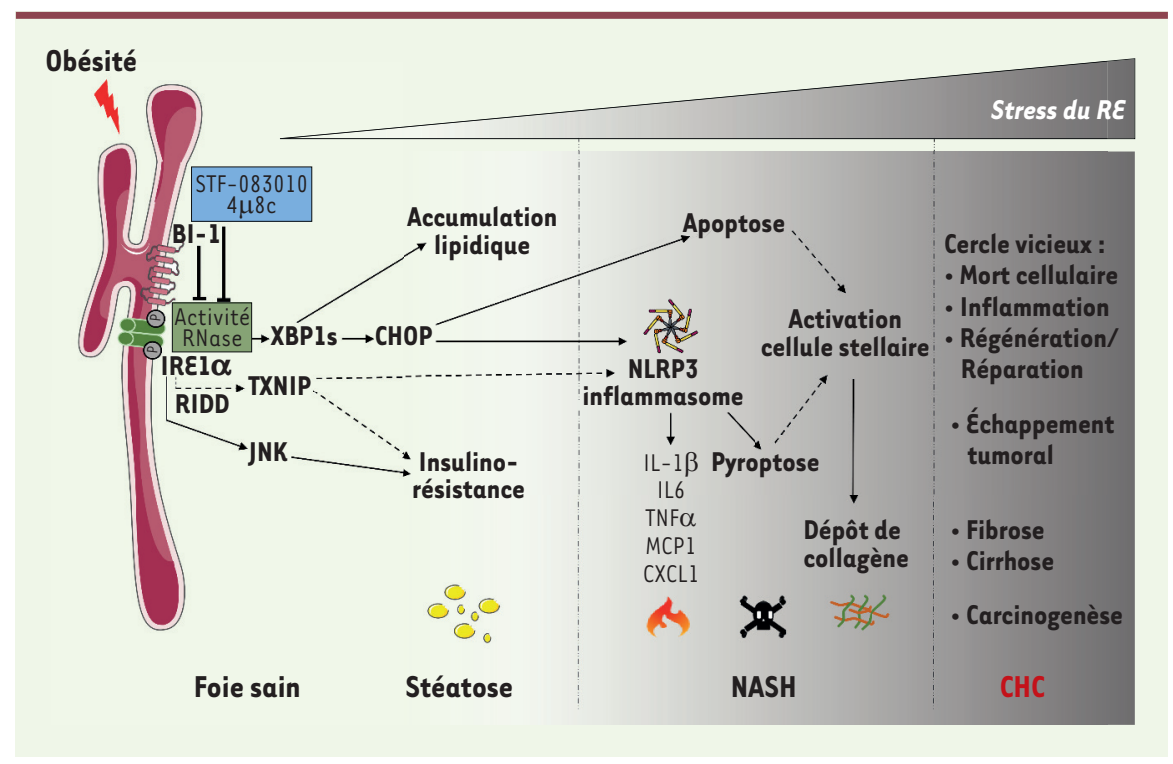

Figure 5. L'inhibition pharmacologique d'IRE1 $\alpha$ : une nouvelle piste thérapeutique. Lors de l'obésité, la voie de signalisation IREl $\alpha$ (inositol-requiring enzyme $1 \alpha$ ) participe à l'installation de la résistance à l'insuline et à l'accumulation intrahépatique des lipides contribuant à l'apparition de la stéatose. Son activation soutenue conduit à l'inflammation et à des processus de mort cellulaire (apoptose et pyroptose), des caractéristiques physiopathologiques de la NASH (stéatohépatite non alcoolique), un facteur à risque du CHC (hépatocarcinome). Le ciblage pharmacologique de l'activité endoribonucléase d'IREl $\alpha$ apparaît être une piste thérapeutique pertinente dans le traitement de la NASH.

de la NASH en inhibant le stress oxydatif, la libération de cytokines inflammatoires et les facteurs induisant la fibrose [36]. Chez des rats présentant une NASH, l'activation pharmacologique de NRF2 réduit significativement la fibrose, faisant de NRF2 une cible thérapeutique potentielle pour le traitement de patients NASH atteints de fibrose hépatique. NRF2 peut également réprimer la transcription de gènes codant des agents pro-oxydants, comme TXNIP qui relie inflammasome et stress oxydant. Dans l'ensemble, le stress du RE intervient dans la régulation positive ou négative du stress oxydatif renforçant l'inflammation et la mort cellulaire. Le stress du RE coopère donc étroitement avec le stress oxydatif dans la pathophysiologie de la NASH.

\section{Stress du RE et cellules immunitaires}

Le foie est également constitué de cellules de l'immunité innée qui, en constituant sa première ligne de défense, participent au maintien de son homéostasie. II s'agit des macrophages résidents du foie (ou cellules de Kupffer), des cellules dendritiques et du couple de cellules NK/NKT (natural killer et natural killer T). Dans la NASH, le nombre de cellules de Kupffer n'est pas modifié mais leur phénotype est altéré. Activées, les cellules de Kupffer s'engagent dans un profil pro-inflammatoire (appelé $\mathrm{Ml}$ ) et il a été montré que la voie de I'UPR, notamment avec $\mid R \varepsilon l \alpha$, joue un rôle dans la polarisation $\mathrm{Ml}$ des macrophages [37-40] $\rightarrow$ ). $(\rightarrow)$ Voir la Nouvelle de A. L'Hermitte et al., $\mathrm{m} / \mathrm{s} \mathrm{n}^{\circ} 11$, novembre 2016, page 1023
De même, une diminution du nombre de cellules NKT est observée au cours de l'obésité et de la NASH, et les cellules présentes montrent un profil pro-inflammatoire (de type Thl). Il a été proposé que lors de l'obésité, l'activation du stress du RE puisse conduire à une diminution de l'expression par les hépatocytes de la molécule CDId, impliquée dans l'activation des cellules NKT, participant au défaut d'activation de ces cellules [41].

Une diminution du nombre de lymphocytes T CD4 ${ }^{+}$et de lymphocytes T régulateurs (Treg) est également observée au cours de la NASH, résultant en une perte d'activité régulatrice de la réponse immuni- taire. Inversement, une activation des lymphocytes $T$ $\mathrm{CD}^{+}$a été rapportée. La voie IREl/XBPl joue un rôle dans l'activation des lymphocytes Treg favorisant I'orientation vers un profil Thl7 inflammatoire et elle est impliquée dans l'activation des lymphocytes T CD8 ${ }^{+}$. Des liens existent donc entre l'activation de l'UPR et les perturbations du système immunitaire [42], qui restent encore à explorer dans la NASH.

Stress du RE et processus de mort cellulaire associés à la NASH

\section{L'apoptose dépendant du stress du RE}

Lorsque les voies de l'UPR ne parviennent pas à résoudre le stress induit par l'accumulation des protéines, les voies activées conduisent à l'apoptose de la cellule. Les voies de I'UPR convergent vers l'activation du facteur de transcription pro-apoptotique CHOP, augmentant l'expression des membres pro-apoptotiques de la famille $\mathrm{BCl}-2$ et conduisant à l'apoptose. CHOP est impliqué dans les mécanismes d'expression de protéines de la famille BH3-only proteins, telles que PUMA, NOXA (ou «dommage» en latin), BIM (bcl-2-interacting mediator of cell death) qui activent directement les membres de la famille $\mathrm{Bcl}-2$ (et/ou répriment les molécules anti-apoptotiques de la même famille) conduisant à l'apoptose. Ces protéines activent, par exemple, directement BAX (Bcl2-associated $X$ protein) et BAK ( $B C l-2$ homologous antagonist/killer), contribuent à la formation de pores au niveau de la mitochondrie, induisant la libération du cytochrome c, l'activation des caspases exécutrices de l'apoptose (caspases-3, -6 , et -7 ) et aboutissant à la mort par apoptose [43] (Figure 4). Ainsi, la voie de l'apoptose mitochondriale 
amplifie les mécanismes pro-apoptotiques qui ont été engagés au niveau des voies de l'UPR. BAX et BAK interagissent également avec le domaine cytosolique d'IREl $\alpha$, ce qui conduit à l'apoptose. Cette interaction semble nécessaire à l'activité d'IREl $\alpha$, la liaison de BI-1 (Bax Inhibitor 1) sur IREl $\alpha$ au site de fixation de BAX et BAK, protégeant de l'apoptose induite par BAX/BAK. La formation du complexe IREl $\alpha$ / TRAF2 permettrait également l'activation de la caspase-12 impliquée dans l'initiation de l'apoptose en lien avec le stress du RE.

L'hypothèse que l'apoptose induite par la réponse au stress du RE dans les cellules hépatiques joue un rôle important dans l'apparition de la NASH est renforcée par la mise en évidence de l'augmentation de marqueurs du stress du RE, notamment CHOP, au sein de biopsies hépatiques de patients NASH [44]. Cette observation a été confirmée par une première étude de notre équipe réalisée chez des patients NASH, qui a révélé que l'expression de CHOP était corrélée au score d'activité des NAFLD et des lésions hépatiques [28]. Les résultats d'une seconde étude réalisée sur des biopsies hépatiques de patients et dans des modèles animaux de NASH, ont suggéré que l'activité RNase d'IREl $\alpha$ jouerait un rôle central dans l'apparition de la maladie [34].

En effet, les souris déficientes pour $B I-1[45,46]$ développent les caractéristiques physiopathologiques de la NASH sous régime gras, et l'inhibition pharmacologique de l'activité RNase d'IREl $\alpha$ corrige la NASH (apoptose, nécroptose, inflammation) chez les souris déficientes pour $B I-1$, mettant en évidence un rôle central de l'activité RNase d'IREl $\alpha$. Une étude réalisée sur des biopsies hépatiques de patients NASH a néanmoins révélé des incohérences quant aux voies de signalisation induites en aval de l'activation de PERK, notamment une activation d'elF2 $\alpha$ mais non de l'axe ATF4-CHOP. De même, pour IREl $\alpha$, la voie JNK serait activée dans la NASH, et XBPl ne serait pas modifié. Confirmant un rôle pro-apoptotique des kinases de la famille JNK dans la pathogenèse de la NASH, les souris déficientes pour JNKl, et nourries avec un régime riche en graisses, sont protégées contre le développement de la résistance à l'insuline hépatique et de la stéatohépatite, notamment en raison de la diminution de l'apoptose des cellules [47].

\section{Lipotoxicité et mort cellulaire}

L'accumulation de lipides dans les foies stéatosiques augmente la lipotoxicité et aggrave les symptômes de la NASH. Les analyses lipidomiques réalisées chez des patients atteints de NASH, ont révélé que le taux d'acides gras libres ( $A G L$ ) sériques qui contribuent davantage aux lésions hépatiques (lipotoxicité) que les triglycérides, était plus important chez ces patients. Chez des souris soumises à un régime riche en graisses, l'inhibition de la synthèse des triglycérides par la DGAT2 (diglyceride acyltransferase 2) entraîne une accumulation d'acides gras libres associée à une élévation du cytochrome P4502\&l (CYP2El), des marqueurs de la peroxydation lipidique/stress oxydatif et de la nécroinflammation lobulaire et de la fibrose [48]. De nombreuses études ont rapporté que les acides gras libres saturés, comme l'acide palmitique ou l'acide stéarique, par opposition aux acides gras libres insaturés, comme l'acide oléique, sont toxiques pour les hépatocytes, activant l'apoptose dépendant du stress du RE, et qu'ils sont impliqués dans la pathogenèse de la NASH. Les rats nourris avec un régime riche en graisses saturées, présentent ainsi une augmentation de l'expression de CHOP et de la caspase-3 active [49]. De même, dans des lignées cellulaires hépatocytaires, l'acide palmitique active spécifiquement les voies PERK et IREl $\alpha$, augmentant ainsi l'expression de CHOP, qui à son tour induit l'expression de DR5 (death receptor 5) conduisant à l'activation de la caspase- 8 et de BID tronquée (tBID). À noter également que l'invalidation de BAX et BAK dans le foie, conduit à l'absence d'apoptose en réponse à un stress du RE, avec une modification de la signalisation de I'UPR [50] (Figure 4). L'activation de la voie IREl $\alpha$ par l'acide palmitique via JNK et/ou CHOP favorise l'activation des membres pro-apoptotiques de la famille $\mathrm{Bcl}-2$ (et/ou répriment les molécules anti-apoptotiques de la même famille) conduisant à l'apoptose.

\section{Cibler le stress du RE dans la NASH : une perspective thérapeutique?}

L'utilisation d'inhibiteurs des protéines senseurs pourraient permettre de diminuer l'activation des voies de mort cellulaire, d'inflammation et les perturbations métaboliques. Ces molécules représenteraient ainsi des perspectives thérapeutiques novatrices.

Plusieurs composés pharmacologiques sont disponibles pour inhiber l'activité RNase d'IREl $\alpha$. Parmi eux, on trouve des inhibiteurs de l'activité RNase, à base de salicylaldéhyde $(4 \mu 8 c$, STF-083010, MKC-3946). Le $4 \mu 8 c$ et le STF-083010 ont donné des résultats prometteurs dans un modèle de NASH [34]. Des composés inhibiteurs de l'activité kinase d'IREl $\alpha$ sont également disponibles (Sunitinib, APy29) mais n'ont pas été testés dans la NASH. Récemment, il a été démontré que la fonction RNase d'IREl $\alpha$ pouvait être inhibée via sa fonction kinase par une nouvelle classe pharmacologique d'inhibiteurs appelés KIRA (kinase-inhibiting rnase attenuators). L'impact de ces composés dans la NASH reste donc à explorer. ASKl est inhibée par le selonsertib, actuellement en essai clinique de phase II chez les patients NASH.

Des inhibiteurs de PERK ont également été développés. Le GSK-265157, un dérivé du GSK-2606414 compétiteur du domaine de fixation de l'ATP, a donné des résultats encourageants dans des modèles murins de cancer hépatique. Cependant, sa sélectivité est questionnée en raison de sa capacité à inhiber RIPKl et la nécroptose. Le séquençage à haut débit a permis de détecter de petits inhibiteurs moléculaires d'ATF6 $\alpha$ appelés céapines qui piègent la protéine dans le $R \varepsilon$, empêchant ATF6 $\alpha$ de se localiser dans le Golgi, bloquant ainsi son activation protéolytique. Les céapines n'ont pas encore été testés dans des modèles murins de NAFLD. 
La sélectivité des nouveaux inhibiteurs d'IREl $\alpha$ et PERK nécessite d'être mieux définie. Au vu des rôles cruciaux d'IREl $\alpha$ et d'XBPI dans le métabolisme des lipides hépatiques, des processus de détoxification et de la survie cellulaire, des potentiels effets hépatotoxiques, il est important d'examiner les éventuels effets secondaires de ces molécules.

Une meilleure compréhension des subtilités de la signalisation UPR chez les patients atteints de NAFLD permettra à plus long terme de stratifier les patients pouvant bénéficier d'une intervention thérapeutique ciblée « à la carte » $[53](\rightarrow)$.

\section{$(\rightarrow)$ Voir la Syn- thèse de $S$. Taouji et $\varepsilon$. Chevet, $m / s n^{\circ}$ 6-7, juin-juillet 2015, page 667}

\section{Conclusion}

L'implication du stress du RE dans la pathophysiologie des complications hépatiques joue un rôle capital, particulièrement dans la transition stéatose-NASH. Étudier le stress du RE permettrait de découvrir de nouvelles cibles thérapeutiques puisqu'il n'existe aucun traitement pharmacologique. Cibler l'activité RNase d'IREl $\alpha$ apparaît comme une perspective thérapeutique pertinente (Figure 5 ). $\diamond$

\section{SUMMARY}

Endoplasmic reticulum stress response and pathogenesis of nonalcoholic steatohepatitis

The incidence of chronic liver disease is constantly increasing, owing to the obesity epidemic. Non-alcoholic fatty liver disease (NAFLD) is currently affecting $20-30 \%$ of the general population and $75-100 \%$ of obese individuals. NAFLD ranges from simple steatosis to damaging non-alcoholic steatohepatitis (NASH), potentially developing into hepatocellular carcinoma. No efficient pharmacological treatment is yet available. During obesity, the hepatic $\varepsilon R$ stress response can arise from extracellular stress (lipids, glucose, cytokines) and from intracellular stress including lipid buildup in the hepatocyte (steatosis), a hallmark of NAFLD. The chronic activation of the hepatic $\varepsilon R$ stress response may be a crucial event in the steatosis-NASH transition, triggering cell death, inflammation and accelerating metabolic disorders. We discuss these aspects and we propose that targeting the $\varepsilon R$ stress response could be effective in treating NAFLD. $\diamond$

\section{LIENS D'INTÉRÊT}

Les auteures déclarent n'avoir aucun lien d'intérêt concernant les données publiées dans cet article.

\section{RéFÉRENCES}

1. Younossi ZM, Koenig AB, Abdelatif D, et al. Global epidemiology of nonalcoholic fatty liver disease-Meta-analytic assessment of prevalence, incidence, and outcomes. Hepatology 2016 ; $64: 73-84$.

2. Piscaglia F, Svegliati-Baroni G, Barchetti A, et al. Clinical patterns of hepatocellular carcinoma in nonalcoholic fatty liver disease: a multicenter prospective study. Hepatology $2016 ; 63: 827-38$.

3. Arrese M, Cabrera D, Kalergis AM, Feldstein AE. Innate Immunity and Inflammation in NAFLD/NASH. Dig Dis Sci 2016 ; 61 : 1294-303.

4. Lebeaupin C, Vallee D, Hazari Y, et al. Endoplasmic reticulum stress signalling and the pathogenesis of non-alcoholic fatty liver disease.J Hepatol 2018 ; 69 : 927-47.

5. Bouchecareilh $M$, Chevet $\varepsilon$. Stress du réticulum endoplasmique : une réponse pour éviter le pIRE. Med Sci (Paris) $2009 ; 25: 281-7$.
6. Cullinan SB, Diehl JA. PERK-dependent activation of Nrf2 contributes to redox homeostasis and cell survival following endoplasmic reticulum stress. J Biol Chem $2004 ; 279: 20108-17$.

7. Harding HP, Zhang $Y$, Zeng $H$, et al. An integrated stress response regulates amino acid metabolism and resistance to oxidative stress. Mol Cell 2003; $11: 619-33$.

8. Adachi Y, Yamamoto K, Okada T, et al. ATF6 is a transcription factor specializing in the regulation of quality control proteins in the endoplasmic reticulum. Cell Struct Funct $2008 ; 33: 75-89$.

9. Yamamoto K, Takahara K, Oyadomari S, et al. Induction of liver steatosis and lipid droplet formation in ATF6alpha-knockout mice burdened with pharmacological endoplasmic reticulum stress. Mol Biol Cell $2010 ; 21$ : 2975-86.

10. Wang JM, Qiu Y, Yang Z, et al. IRElalpha prevents hepatic steatosis by processing and promoting the degradation of select microRNAs. Sci Signal $2018 ; 11$

11. Foufelle F, Ferre $P$. La réponse UPR : son rôle physiologique et physiopathologique. Med Sci (Paris) 2007 ; 23 : 291-6.

12. Ozcan U, Cao $Q$, Yilmaz $\varepsilon$, et al. Endoplasmic reticulum stress links obesity, insulin action, and type 2 diabetes. Science 2004 ; 306 : 457-61.

13. Gregor MF, Yang L, Fabbrini $\varepsilon$, et al. Endoplasmic reticulum stress is reduced in tissues of obese subjects after weight loss. Diabetes $2009 ; 58: 693-700$.

14. Lake AD, Novak P, Hardwick RN, et al. The adaptive endoplasmic reticulum stress response to lipotoxicity in progressive human nonalcoholic fatty liver disease. Toxicol Sci 2014 ; 137 : 26-35.

15. Yoshiuchi K, Kaneto $H$, Matsuoka TA, et al. Direct monitoring of in vivo $\varepsilon R$ stress during the development of insulin resistance with $E R$ stress-activated indicator transgenic mice. Biochem Biophys Res Commun 2008 ; 366 : 545-50.

16. Kammoun HL, Chabanon H, Hainault I, et al. GRP78 expression inhibits insulin and $\varepsilon R$ stress-induced SREBP-lc activation and reduces hepatic steatosis in mice. J Clin Invest 2009 ; 119: 1201-15.

17. Gorden DL, Myers DS, Ivanova PT, et al. Biomarkers of NAFLD progression: a lipidomics approach to an epidemic. J Lipid Res 2015; 56 : 722-36.

18. Farese RV, Jr., Zechner R, Newgard CB, Walther TC. The problem of establishing relationships between hepatic steatosis and hepatic insulin resistance. Cell Metab $2012 ; 15: 570-3$.

19. Flamment M, Foufelle F. Stéatose hépatique et stress du réticulum endoplasmique : une histoire de phospholipides. Med Sci (Paris) 2012 ; 28 : 13-5.

20. Volmer R, van der Ploeg K, Ron D. Membrane lipid saturation activates endoplasmic reticulum unfolded protein response transducers through their transmembrane domains. Proc Natl Acad Sci USA $2013 ; 110$ : 4628-33.

21. Patterson RE, Kalavalapalli $S$, Williams CM, et al. Lipotoxicity in steatohepatitis occurs despite an increase in tricarboxylic acid cycle activity. Am J Physiol Endocrinol Metab 2016 ; 310 : E484-94.

22. Patouraux $S$, Rousseau $D$, Bonnafous $S$, et al. CD44 is a key player in nonalcoholic steatohepatitis. J Hepatol 2017 ; 67 : 328-38.

23. Feldstein AE, Canbay A, Angulo P, et al. Hepatocyte apoptosis and fas expression are prominent features of human nonalcoholic steatohepatitis. Gastroenterology $2003 ; 125: 437-43$.

24. Deng J, Lu PD, Zhang $Y$, et al. Translational repression mediates activation of nuclear factor kappa B by phosphorylated translation initiation factor 2 . Mol Cell Biol 2004 ; 24 : 10161-8.

25. Luedde T, Schwabe RF. NF-kappaB in the liver: linking injury, fibrosis and hepatocellular carcinoma. Nat Rev Gastroenterol Hepatol $2011 ; 8$ : 108-18.

26. Willy JA, Young SK, Stevens JL, et al. CHOP links endoplasmic reticulum stress to NF-kappaB activation in the pathogenesis of nonalcoholic steatohepatitis. Mol Biol Cell $2015 ; 26: 2190-204$.

27. Yoneda M, Mawatari H, Fujita K, et al. High-sensitivity C-reactive protein is an independent clinical feature of nonalcoholic steatohepatitis (NASH) and also of the severity of fibrosis in NASH. J Gastroenterol $2007 ; 42: 573-82$.

28. Lebeaupin C, Proics $\varepsilon$, de Bieville CH, et al. ER stress induces NLRP3 inflammasome activation and hepatocyte death. Cell Death Dis $2015 ; 6$ : e1879.

29. Stienstra R, van Diepen JA, Tack CJ, et al. Inflammasome is a central player in the induction of obesity and insulin resistance. Proc Natl Acad Sci USA 2011 ; 108 : 15324-9.

30. Galluzzi L, Vitale I, Abrams JM, et al. Molecular definitions of cell death subroutines: recommendations of the nomenclature committee on cell death 2012. Cell Death Differ $2012 ; 19$ : 107-20.

31. Wree A, Eguchi A, McGeough MD, et al. NLRP3 inflammasome activation results in hepatocyte pyroptosis, liver inflammation, and fibrosis in mice. Hepatology 2014 ; 59 : 898-910. 


\section{RÉFÉRENCES}

32. Mridha AR, Wree A, Robertson AAB, et al. NLRP3 inflammasome blockade reduces liver inflammation and fibrosis in experimental NASH in mice.J Hepatol $2017 ; 66: 1037-46$.

33. Lerner AG, Upton JP, Praveen PV, et al. IRElalpha induces thioredoxin-interacting protein to activate the NLRP3 inflammasome and promote programmed cell death under irremediable ER stress. Cell Metab $2012 ; 16: 250-64$.

34. Lebeaupin C, Vallee D, Rousseau D, et al. Bax inhibitor-1 protects from nonalcoholic steatohepatitis by limiting inositol-requiring enzyme 1 alpha signaling in mice. Hepatology 2018 ; $68: 515-32$.

35. Zmijewski JW, Banerjee $\mathrm{S}$, Bae $\mathrm{H}$, et al. Exposure to hydrogen peroxide induces oxidation and activation of AMP-activated protein kinase. J Biol Chem 2010 ; 285 : 33154-64.

36. Okada K, Warabi $\varepsilon$, Sugimoto $H$, et al. Nrf2 inhibits hepatic iron accumulation and counteracts oxidative stress-induced liver injury in nutritional steatohepatitis. J Gastroenterol 2012 ; 47 : 924 35.

37. Shan B, Wang X, Wu Y, et al. The metabolic ER stress sensor IRElalpha suppresses alternative activation of macrophages and impairs energy expenditure in obesity. Nat Immunol 2017; 18 519-29.

38. Reverendo M, Mendes A, Argüello RJ, et al. At the crossway of $\varepsilon R$-stress and proinflammatory responses. The FEBS Journal $2019 ; 286: 297-310$.

39. Cassard-Doulcier A-M, Perlemuter G. Inflammation hépatique liée à l’obésité (NASH). Oléagineux Corps gras Lipides $2011 ; 18: 21-6$.

40. L'Hermitte A, Pham S, Cadoux M, Couty J-P. Hépatopathies stéatosiques non alcooliques. Med/Sci (Paris) $2016 ; 32: 1023-6$

41. Yang L, Jhaveri R, Huang J, et al. Endoplasmic reticulum stress, hepatocyte CDld and NKT cell abnormalities in murine fatty livers. Lab Invest $2007 ; 87: 927-37$.

42. Szpigel A, Hainault I, Carlier A, et al. Lipid environment induces $E R$ stress, TXNIP expression and inflammation in immune cells of individuals with type 2 diabetes. Diabetologia $2018 ; 61: 399$ 412

43. Deniaud A, Sharaf el dein 0, Maillier $\varepsilon$, et al. Endoplasmic reticulum stress induces calciumdependent permeability transition, mitochondrial outer membrane permeabilization and apoptosis. Oncogene $2008 ; 27: 285-99$.

44. Gonzalez-Rodriguez A, Mayoral R, Agra N, et al. Impaired autophagic flux is associated with increased endoplasmic reticulum stress during the development of NAFLD. Cell Death Dis $2014 ; 5$ : el179
45. Bailly-Maitre B, Belgardt BF, Jordan SD, et al. Hepatic Bax inhibitor-1 inhibits IRElalpha and protects from obesity-associated insulin resistance and glucose intolerance. J Biol Chem 2010 ; 285 : 6198-207.

46. Bailly-Maitre B, Fondevila C, Kaldas F, et al. Cytoprotective gene bi- 1 is required for intrinsic protection from endoplasmic reticulum stress and ischemia-reperfusion injury. Proc Natl Acad Sci USA 2006 ; 103 : 2809-14.

47. Schattenberg JM, Singh R, Wang Y, et al. JNK1 but not JNK2 promotes the development of steatohepatitis in mice. Hepatology $2006 ; 43: 163-72$.

48. Yamaguchi K, Yang L, McCall S, et al. Inhibiting triglyceride synthesis improves hepatic steatosis but exacerbates liver damage and fibrosis in obese mice with nonalcoholic steatohepatitis. Hepatology $2007 ; 45$ : 1366 74

49. Wang D, Wei Y, Pagliassotti MJ. Saturated fatty acids promote endoplasmic reticulum stress and liver injury in rats with hepatic steatosis. Endocrinology $2006 ; 147: 943-51$.

50. Hetz C, Bernasconi P, Fisher J, et al. Proapoptotic BAX and BAK modulate the unfolded protein response by a direct interaction with IRElalpha. Science $2006 ; 312: 572-6$

51. Rieusset J. Endoplasmic reticulum-mitochondria calcium signaling in hepatic metabolic diseases. Biochim Biophys Acta Mol Cell Res 2017; 1864 $865-76$.

52. $\mathrm{Xu} C, \mathrm{Xu}$ W, Palmer AE, Reed JC. BI-1 regulates endoplasmic reticulum $\mathrm{Ca}^{2+}$ homeostasis downstream of $\mathrm{Bcl}-2$ family proteins. J Biol Chem $2008 ; 283$ : 11477-84.

53. Taouji $S$, Chevet $\varepsilon$. Modulation pharmacologique de la réponse au stress du réticulum endoplasmique : potentiel thérapeutique en cancérologie. Med Sci (Paris) $2015 ; 31: 667-73$.

\section{TIRÉS À PART}

B. Bailly-Maitre

P ossédées du Malin au Moyen-Âge, les sorcières hystériques sont vouées au bûcher. Enfermées au XvII siècle, maltraitées, elles rejoignent la Cour des Miracles de l'Hospice de la Vieillesse-Femmes à la Salpêtrière... Jusqu'à ce que le Dr JeanMartin Charcot (1825-1893) mène le combat qui transforme l'ancien hospice en hôpital : l'École de la Salpêtrière de

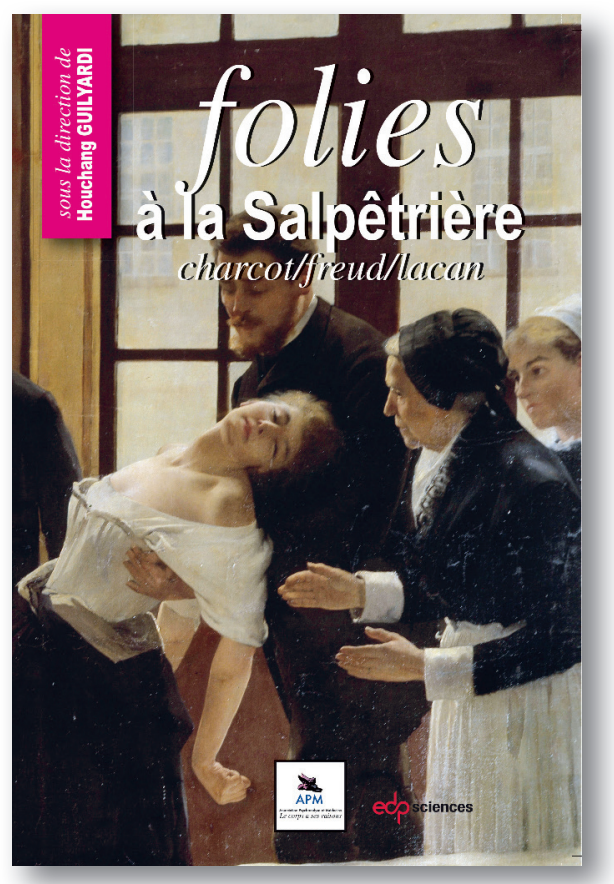

ISBN : 978-2-7598-1268-4
240 pages Paris est née, qui devient lieu de recherche, d'enseignement et de soins, de renommée internationale.

Jean Martin Charcot n'a pas bonne presse, et pourtant... Hystérie et folie traversent les siècles, prenant les formes de «l'air du temps ».

De l'utérus migrateur d'Hippocrate aux recherches neurologiques de Charcot. Du désir inconscient avec Freud à la jouissance du parlêtre chez Lacan... C'est à cette traversée historique et conceptuelle que nous convie cet ouvrage.

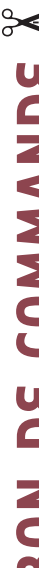

UU À retourner à EDP Sciences, 17 avenue du Hoggar, 91944 Les Ulis Cedex, France

Tél. : 0149856069 - Fax : 0149850345 - $\varepsilon$-mail : francois.flori@edpsciences.org

NOM : $\quad$ Prénom

Adresse : ...

Code postal :

Pays :

Fonction :

Folies à la Salpêtrière : $20 €+3 €$ de port $=23 € \pi C$

en

$\neg$ Par chèque, à l'ordre de EDP Sciences

$\square$ Par carte bancaire : $\quad \square$ Visa $\square$ Eurocard/Mastercard

Carte $n^{\circ} \sqcup|\perp| \perp|\perp| \perp|\downarrow| \perp|\perp| \sqcup|\perp|$

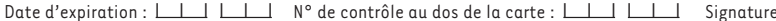

